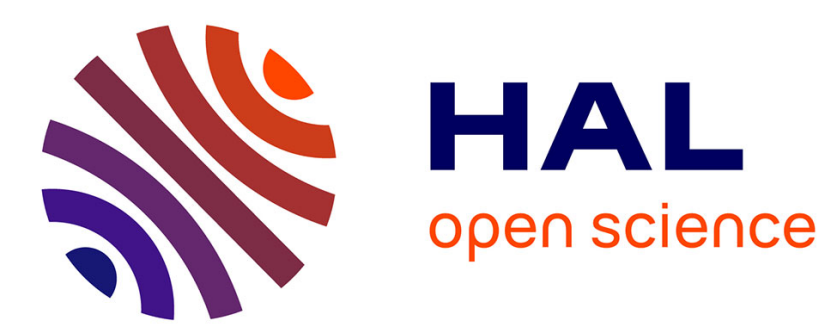

\title{
When the exception becomes the rule: The disappearance of limiting similarity in the Lotka-Volterra model
}

György Barabás, Géza Meszéna

\section{- To cite this version:}

György Barabás, Géza Meszéna. When the exception becomes the rule: The disappearance of limiting similarity in the Lotka-Volterra model. Journal of Theoretical Biology, 2009, 258 (1), pp.89. 10.1016/j.jtbi.2008.12.033 . hal-00554558

\section{HAL Id: hal-00554558 \\ https://hal.science/hal-00554558}

Submitted on 11 Jan 2011

HAL is a multi-disciplinary open access archive for the deposit and dissemination of scientific research documents, whether they are published or not. The documents may come from teaching and research institutions in France or abroad, or from public or private research centers.
L'archive ouverte pluridisciplinaire HAL, est destinée au dépôt et à la diffusion de documents scientifiques de niveau recherche, publiés ou non, émanant des établissements d'enseignement et de recherche français ou étrangers, des laboratoires publics ou privés. 


\section{Author's Accepted Manuscript}

When the exception becomes the rule: The disappearance of limiting similarity in the Lotka-Volterra model

György Barabás, Géza Meszéna

PII:

S0022-5193(09)00003-4

DOI: doi:10.1016/j.jtbi.2008.12.033

Reference: YJTBI 5420

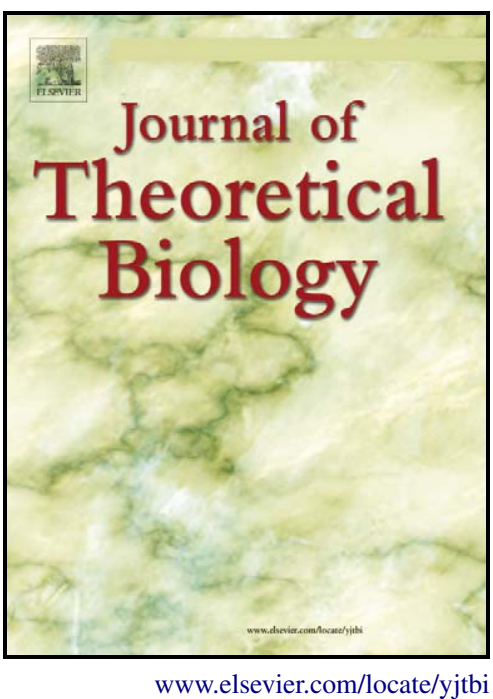

To appear in: Journal of Theoretical Biology

Received date: $\quad 8$ July 2008

Revised date: $\quad 16$ December 2008

Accepted date: 29 December 2008

Cite this article as: György Barabás and Géza Meszéna, When the exception becomes the rule: The disappearance of limiting similarity in the Lotka-Volterra model, Journal of Theoretical Biology (2009), doi:10.1016/j.jtbi.2008.12.033

This is a PDF file of an unedited manuscript that has been accepted for publication. As a service to our customers we are providing this early version of the manuscript. The manuscript will undergo copyediting, typesetting, and review of the resulting galley proof before it is published in its final citable form. Please note that during the production process errors may be discovered which could affect the content, and all legal disclaimers that apply to the journal pertain. 


\title{
When the exception becomes the rule: the disappearance of limiting similarity in the Lotka-Volterra model
} \\ György Barabás ${ }^{1} \&$ Géza Meszéna \\ Department of Biological Physics, Eötvös University \\ Pázmány Péter sétány 1A, H-1117 Budapest, Hungary \\ phone: 36-1-372-2795, fax: 36-1-372-2757 \\ email: dysordys@umich.edu,geza.meszena@elte.hu
}

\begin{abstract}
We investigate the transition between limiting similarity and coexistence of a continuum in the competitive Lotka-Volterra model. It is known that there exist exceptional cases in which, contrary to the limiting similarity expectation, all phenotypes coexist along a trait axis. Earlier studies established that the distance between surviving phenotypes is in the magnitude of the niche width $2 \sigma$ provided that the carrying capacity curve differs from the exceptional one significantly enough. In this paper we studied the outcome of competition for small perturbations of the exceptional (Gaussian) carrying capacity. We found that the average distance between the surviving phenotypes goes to zero when the perturbation vanishes. The number of coexisting species in equilibrium is proportional to the negative logarithm of the perturbation. Nevertheless, the niche width provides a good order of magnitude for the distance between survivors if the perturbations are larger than $10 \%$. Therefore, we conclude that limiting similarity is a good framework of biological thinking despite the lack of an absolute lower bound of similarity.
\end{abstract}

Keywords: competitive exclusion, continuous coexistence, niche width

\footnotetext{
${ }^{1}$ corresponding author
} 


\section{${ }_{19} \quad 1$ Introduction}

${ }_{20}$ The species packing problem is one of the oldest issues of mathematical ecology. Based on the

21 investigation of the Lotka-Volterra competition model, MacArthur and Levins (1967) proposed

22 that species should not be too similar if they are to coexist (limiting similarity). The minimal

23 difference was assumed to be related to the niche width $2 \sigma$ of the species, i.e. to the width of their

${ }_{24}$ resource utilization function. This insight turned out to be useful in interpreting empirical data in

25 simple cases of resource competition (Schluter, 1982; Grant and Schluter, 1984; Grant, 1999).

${ }_{26}$ Unfortunately, the naïve version of limiting similarity was not confirmed by further theoretical

27 studies. May and MacArthur (1972); May (1973) found that the minimal trait difference can be

${ }_{28}$ arbitrarily small if the carrying capacities are properly tuned. Even worse, Roughgarden (1979)

29 (p. 534-536) demonstrated that even a continuum of phenotypes may coexist in the Lotka-Volterra

30 model. These results were seen by many as the end of the road to limiting similarity (Rosenzweig,

31 1995; Maynard Smith and Szathmáry, 1995).

32 However, Abrams (1983) proposed a different viewpoint. As there is no such thing as an absolute

33 lower limit of allowed similarity, one should instead study the relationship between similarity and the

34 likelihood of coexistence. In the competitive Lotka-Volterra model it is easy to see that increasing

35 similarity shrinks the parameter range allowing for coexistence (May, 1973; Vandermeer, 1975). This

36 relationship was formally proven in a model-independent way by Meszéna et al. (2006). Coexistence

${ }_{37}$ of similars is not impossible, but it is sensitive to external perturbations and is therefore improbable.

${ }_{38}$ This way, the idea of limiting similarity can be rescued as a basis of biological thinking, despite the

39 lack of an absolute lower bound of similarity.

$40 \quad$ In line with the perturbation approach of Meszéna et al. (2006), Gyllenberg and Meszéna (2005)

${ }_{41}$ demonstrated that continuous coexistence, like the one in Roughgarden (1979)'s model, is always

42 structurally unstable, i.e. it can be destroyed by an arbitrarily small perturbation. This result is

43 also independent of the specific model (see Sasaki and Ellner, 1995; Sasaki, 1997, for earlier, related 
44 studies).

45 With this background, Szabó and Meszéna (2006) reinvestigated the Lotka-Volterra competition 46 model and numerically reproduced the cases of continuous coexistence as well as limiting similarity.

47 Provided that the competition function is a Gaussian, the Gaussian carrying capacity represents 48 the special situation allowing coexistence on the whole continuum. Any significant departure from 49 the Gaussian shape of the carrying capacity resulted in a clear discretization of the coexisting types. 50 With reassuring resemblance to the original idea of MacArthur and Levins (1967), the typical trait 51 difference between the neighbouring types is in the order of magnitude of, and is proportional to, 52 the niche width $2 \sigma$.

${ }_{53}$ Here we continue this investigation by studying the transition between continuous coexistence 54 and limiting similarity. We know from the analytical result of Gyllenberg and Meszéna (2005) that ${ }_{55}$ an arbitrarily small perturbation of the continuous case results in a discrete distribution. Can we 56 also conclude that the $2 \sigma$ prediction for the neighbour-distance is also applicable after an arbitrarily 57 small departure from the continuous case? Or will the distance increase gradually from zero? In the latter case, how much perturbation is needed to ensure the validity of the classical picture?

\section{${ }_{59} 2$ Model definition}

60 Following MacArthur and Levins (1967), the Lotka-Volterra model describing competition for a

${ }_{61}$ resource continuum is investigated. Each species is characterized by a single continuous trait de62 scribing the species' resource optimum.

63 For $L$ different species, the population dynamics is specified as

$$
\frac{d n_{i}(t)}{d t}=\left(K\left(x_{i}\right)-\sum_{j=1}^{L} a\left(x_{i}, x_{j}\right) n_{j}(t)\right) n_{i}(t) \quad(i=1, \ldots, L),
$$

${ }_{64}$ where $n_{i}(t)$ and $x_{i}$ are the density and the phenotype of the $i$ th species, respectively. It reads as

$$
\frac{d n(x, t)}{d t}=\left(K(x)-\int a(x, y) n(y, t) d y\right) n(x, t)=0
$$


65

for a continuum of phenotypes. (Note that the concepts of phenotype and species are equivalent in a model of clonal inheritance.)

The competition function is specified to be a Gaussian of the trait difference:

$$
a\left(x_{i}, x_{j}\right)=\exp \left(-\frac{\left(x_{i}-x_{j}\right)^{2}}{2 \sigma^{2}}\right) .
$$

As $a(x, x)=1$ for all $x$, the equilibrium population size of a species, when alone, is $K(x)$. Therefore, $K(x)$ is referred to as the carrying capacity function. It is assumed to have the Gaussian form

$$
K(x)=\exp \left(-\frac{x^{2}}{2 \omega^{2}}\right)
$$

as the reference case. Then for $\omega>\sigma$ the continuous distribution

$$
n(x)=\frac{\omega}{\sqrt{2 \pi \sigma^{2}\left(\omega^{2}-\sigma^{2}\right)}} \exp \left(-\frac{x^{2}}{2\left(\omega^{2}-\sigma^{2}\right)}\right)
$$

of the phenotypes satisfies the equilibrium equation

$$
K(x)-\int a(x, y) n(y, t) d y=0
$$

for the continuous case (Roughgarden, 1979; Szabó and Meszéna, 2006).

We will modify the reference form of the carrying capacity by a perturbing function $f(x)$ as

$$
K(x)=\exp \left(-\frac{x^{2}}{2 \omega^{2}}\right)+\varepsilon f(x)
$$

where the parameter $\varepsilon$ is used to tune the strength of perturbation. As proven in Gyllenberg and Meszéna (2005), for an appropriate choice of the perturbing function, an arbitrarily small $\varepsilon$ destroys the possibility of the coexistence of a whole continuum of phenotypes.

For the simulations the interval $x \in[-1,1]$ was divided into 1001 partitions of equal length. We integrated the so discretized version of Eq. (2) by the Euler method with $\Delta t=1.6$. Our initial conditions at $t=0$ were $n(x, t)=0.01$ for all $x \in[-1,1]$. The simulations were terminated when the number of coexisting species equlibrated. 
In order to be able to determine more precisely when equilibrium was reached, our program

84 to zero. In our simulations this threshold was $n \leq 10^{-6}$. Second, we recorded the number of ${ }_{85}$ phenotypes (out of $L=1001$ ) with nonzero density every 1000 iterations (a typical run of the ${ }_{86}$ program consisted of a few million cycles, or even more for very small perturbations). We could get ${ }_{87}$ a good guess on how far we were from reaching equilibrium, since the convergence of the number 88 of phenotypes with nonzero density (that is, the number of surviving species) to a certain value marked the point from where nothing interesting would happen. By watching this convergence 90 diagram we were able to stop the running of the program when it became necessary.

\section{${ }_{91} \quad 3 \quad$ Results}

${ }_{92}$ The simplest choice for the perturbation function $f(x)$ is

$$
f(x)=\hat{\delta}(x)
$$

${ }_{93}$ where $\hat{\delta}(x)$ is a kind of characteristic function: it is equal to 1 at $x=0$, zero elsewhere. It can be 94 considered as a small $\xi$ limit of $\exp \left(-x^{2} /\left(2 \xi^{2}\right)\right)$ and should not be confused with the Dirac delta 95 function which has an infinite value at $x=0$ (this characteristic function is very convenient for 96 numerical investigations, but one should be careful about a naïve analytical application to Eq. (2) ${ }_{97}$ as the $\xi \rightarrow 0$ limit may lead to problems).

The left pane of Fig. 1 presents the equilibrium solution with this type of perturbation. One can observe the discreteness of species distribution, as predicted by the theory. A very simple mechanism, which is easy to follow by observing the simulations, leads to this discretization. Without the perturbation, the whole range of phenotypes could coexists. However, the central peak of $K$ gives a distinct advantage of the phenotype at $x=0$. Increased density of this population results in 
heightened competition against the similar types. Therefore, species die out in a phenotype range around the species preferred by the perturbation.

The right pane shows the dependence of the number of surviving phenotypes $m$ on the perturbation amplitude $\varepsilon$. It can be fitted very well with the function

$$
m=\alpha-\beta \ln \varepsilon .
$$

One may observe that $m$ diverges for small perturbations, just as it should. However, for $\varepsilon>0.1$ the dependence is weak because the logarithm function barely changes at all in that regime. The number of coexisting species is essentially independent of the perturbation, provided that the perturbation (i.e. $\varepsilon$ ) is not very small. This $\varepsilon$-dependence is also obviously linear when $m$ is considered as a function of $\ln \varepsilon$. The two parameters $\alpha$ and $\beta$ therefore correspond to the parameters of a linear fit. Thus it was convenient to determine these parameters in terms of the above linearity.

As the discretization proceeds outward from the central peak, we used the following trick for counting the coexisting species. Instead of waiting until a perfect equilibrium was reached along the whole interval, the simulation was run until the first few survivors around the perturbation peak were built up. Then, we measured the distance in phenotype between the survivor at the point of the perturbation and its nearest neighbour and divided the total phenotype interval $[-1,1]$ by this distance to obtain the number of surviving phenotypes. Since the survivors are more or less evenly spaced, as one can check on the left pane of the Figure, this method provides just as good results as the straightforward peak counting when $\varepsilon$ goes to zero.

To check whether this result is an artefact of the non-smooth nature of the perturbing function, we repeated the simulations with

$$
f(x)=\exp \left(-x^{2} /\left(2 \xi^{2}\right)\right)
$$

23 using a finite $\xi$ that was smaller than $\sigma$. The results are exactly the same as before (Fig. 2). That is, a narrow peak of perturbation destroys continuous coexistence just like the infinitely narrow one. 
Note that the linear nature of the equilibrium equation (7) implies that continuous coexistence exists for $\xi>\sigma$ with the phenotype-distribution

$$
n(x)=\frac{\omega}{\sqrt{2 \pi \sigma^{2}\left(\omega^{2}-\sigma^{2}\right)}} \exp \left(-\frac{x^{2}}{2\left(\omega^{2}-\sigma^{2}\right)}\right)+\varepsilon \frac{\xi}{\sqrt{2 \pi \sigma^{2}\left(\xi^{2}-\sigma^{2}\right)}} \exp \left(-\frac{x^{2}}{2\left(\xi^{2}-\sigma^{2}\right)}\right) .
$$

The possibility for continuous coexistence disappears when $\xi<\sigma$.

This behavior becomes understandable when one consider that the equilibrium distribution of continuous coexistence is to be calculated from the equilibrium equation (7) by deconvolution (Gyllenberg and Meszéna, 2005). That procedure breaks down when the carrying capacity function has higher significant Fourier components than the competition kernel does. That is, discretization is related to the changes of the carrying capacity function that are sharper than of the competition kernel. While we did not investigate this in detail, it seems that coexistence shrinks immediately to the equivalent of $\xi=0$ when $\xi$ becomes less than $\sigma$.

A similar analysis to the single-peak case with similar results is presented in Fig. 3 for an asymmetrically located perturbing peak, i.e. for

$$
f(x)=\hat{\delta}\left(x-x_{0}\right)
$$

Here the same species counting method was applied as before with using the average of the distances to the two nearest neighbours. Looking at Fig. 3 one may think this method would not work here since the distance between the "privileged" phenotype and its nearest neighbours is obviously greater than the rest of the distances. That is true, but only because the perturbation in this case is very large (it is in the order of magnitude of the original carrying capacity). For small perturbations $(\varepsilon \lesssim 0.1)$ the distances equalize. Therefore this method is actually no worse than the one discussed in the previous case.

Fig. 4 represents the results for the two-peaked perturbation

$$
f(x)=\hat{\delta}\left(x+x_{0}\right)+\hat{\delta}\left(x-x_{0}\right) .
$$


In this case we have more than one "seed" of discretization which, therefore, proceeds much faster.

The species were directly counted after full convergence.

As we learned above, discretization is related to the sharp changes of the carrying capacity function, i.e. to the high Fourier components of the perturbation. To get a balanced picture it is necessary to study a perturbation that is somehow mid-way between being smooth and rough.

From this reason, following Szabó and Meszéna (2006), we study a fractal-like perturbing function:

$$
f(x)=\exp \left(-\frac{\left(x-x_{0}\right)^{2}}{2 \omega^{2}}\right) \cdot\left(\sum_{j=1}^{M} j^{-\eta} \cos \left(j x+\varphi_{j}\right)\right) .
$$

Here $\eta=1.5$ is the critical exponent determining the mixing ration of the different Fourier components. The $\varphi_{j}$ s are random phases and $M=1001$ is the number of partitions of the phenotype axis.

The qualitative conclusions are the same in all cases. The surviving species are distanced roughly according to the niche-width except when we are very close to the structurally unstable situation where $\varepsilon=0$.

Reaching equilibrium takes longer and longer time with decreasing perturbation. Could it be that the higher number of survivors for smaller perturbation is just an artifact of not waiting long enough to allow competitive exclusion to proceed? In Fig. 6 we present a comparison between two equilibrated population distributions for different perturbation amplitudes. Here the perturbation function

$$
f(x)=\sum_{j=1}^{20} \hat{\delta}(x-1+0.1 j)
$$

was applied. The results demonstrate clearly that extra species are present in equilibrium for small perturbations.

The fitting parameter $\alpha$ gives the number of coexisting populations for $\varepsilon=1$. It also provides a reasonable approximation of $m$ for any value of $\varepsilon$ above 0.1 . The average distance between the nearest neighbours equals to $\Delta x=2 / \alpha$. The dependence of the so-calculated $\Delta x$ on the $\sigma$ 
parameter is presented on Fig. 7. One can observe strict proportionality between the two variables.

The value $\delta=2$ of the proportionality constant would correspond to a precise $2 \sigma$ rule. While the real $\delta$ differs from this value, its order of magnitude allow us to consider the species separation by niche width as a good rule of thumb. Note that $\delta$ becomes smaller for the fractal case, presumably because smoother perturbations allow for denser packing.

\section{Discussion}

Here we contributed to the understanding of the long-standing issue of species packing by analyzing the transitional regime between continuous coexistence and limiting similarity in the competitive Lotka-Volterra model. Analytical studies have made it clear already that continuous coexistence is structurally unstable (Gyllenberg and Meszéna, 2005), but a kind of limiting similarity is the generically expected behaviour (Meszéna et al., 2006). However, these results are far from sufficient to guiding biological intuition properly. Is limiting similarity a solid ground of biological thinking, or a shaky one, after all?

This question cannot be put to rest without combining the analytical insights with extensive studies of specific models. As the Lotka-Volterra model is the classical workhorse of these studies, it is a meaningful starting point. In this context Szabó and Meszéna (2006) demonstrated that the niche-width $2 \sigma$ is a good guess for the typical distance between neighbouring surviving species provided that we are far from the situation of continuous coexistence. Here we studied the transition regime.

As continuous coexistence is structurally unstable (i.e. it can be destroyed by an arbitrarily small perturbation of the model definition) one could conjecture that an arbitrarily small departure from this exceptional case would lead us to the "roughly $2 \sigma$ " regime. In this case it would be expected that the transition to continuous coexistence consists of slower and slower relaxation to 
the final distribution. Our simulations demonstrated that this is not the case. The real transition goes through denser and denser discrete equilibrium distributions until the continuous distribution is reached as a limit at the zero value of $\varepsilon$.

This conclusion is in line with the overall picture of limiting similarity. It was demonstrated early on for two, or three, species in the Lotka-Volterra model that increasing similarity narrows the parameter range allowing them to coexist (May and MacArthur, 1972; May, 1973). Higher similarity is allowed, if we are satisfied with a more parameter-sensitive coexistence. Abrams (1983) observed this "similarity-coexistence relationship" to be quite general, while Meszéna et al. (2006) proved it in a model-independent way (see also Szilágyi and Meszéna, 2008, 2009, in prep. about generalizations for structured populations and fluctuations).

On a first glance, the possibility for the coexistence of a continuum of species seems to be surprising. Nevertheless, it is evident that any predefined set of species can be made coexisting by properly tuning their $K$ values. Even more is true: by tuning the $K$ s one can arrange coexistence with any predefined density distribution. Specifically, if the competition function in the LotkaVolterra model is chosen to be Gaussian and the required species distribution is also a Gaussian, then the carrying capacity curve must also be a Gaussian, calculated as a convolution of the other two functions. Then this Gaussian carrying capacity is the one that, exceptionally, supports continuous coexistence with that specific distribution. However, as there are species arbitrarily close to each other, there exists an arbitrarily small perturbation of the carrying capacity curve after which the continuum of coexisting phenotypes no longer exist. Nevertheless, the Gaussian nature of the functions plays no specific role here: the cases of exceptional continuous coexistence could be constructed from other functions as well.

These arguments consider the robustness of the existence of the coexisting fixed point against the perturbation of the model. The dynamical stability of the fixed point (if it exists) is a separate question. From this point of view the shape of the competition function does matter. Pigolotti 
et al. $(2007,2008)$ demonstrated that continuous coexistence in the Lotka-Volterra model is dynamically stable only if the competition function is positive definite, i.e. if its Fourier transform is positive everywhere. From this point of view the Gaussian shape is a borderline case. If a different competition function does not meet this criterion, then the continuous coexistence, constructed in the way mentioned above, will be unstable. This possibility further strengthens the conclusion that the continuous coexistence is not expected to be an observed behaviour.

It is obvious that any extinction threshold sets a limit to similarity in itself (May and MacArthur, 1972; Pigolotti et al., 2007). An infinitely dense assembly of finite populations would consist of infinitely many individuals, a contradiction. In our simulations we set the extinction threshold so low that it did not affect our results. We had to discretize the phenotype axis for practical reasons. Then, it was possible to choose the extinction threshold so low that allowed the dense packing determined by the discretization. Effectively, we studied the limit of similarity without the extinction threshold.

Note also the contradictory nature of postulating a phenotype-continuum together with a separate threshold for each phenotype. Realistically, any kind of Alle effect should consider the similar types together - or the phenotype variable is not a complete characterization of the populations.

Beyond the qualitative conclusions, a clear quantitative picture emerged: the number of survivors is a linear function of the negative logarithm of the perturbation size. This result seems to be fairly general. The very same logarithmic rule (9) emerged for all kinds of perturbing functions we tested, from the simplest possible single peak to the most complex fractal function. In accordance with the continuous coexistence case, the function (9) goes to infinity when $\varepsilon \rightarrow 0$. However, the transition from discrete to continuous happens quite fast, but still in a continuous way, for small $\varepsilon s$. For $\varepsilon \gtrsim 0.1$ one may rely on the intuition of a "niche width".

A numerical study of such a simplified model as the Lotka-Volterra acquires its relevance from the hope that the results are more general than the specific assumptions. Having the general 
analytic theories both for continuous coexistence (Gyllenberg and Meszéna, 2005) and for limiting

similarity (Meszéna et al., 2006) motivates us to conjecture the genericity of the transition regime between them as well. Unfortunately, we failed to find an analytic theory behind the observed logarithmic dependence. It is quite easy to derive analytic results for coexistence of two, or a few, species in the Lotka-Volterra model. However, we considered the coexistence of phenotypes, the number of which goes to infinity in the $\varepsilon \rightarrow 0$ limit. Lacking the analytic insight, it is impossible to assess the validity of our conjecture without studying further models numerically.

While further analysis of the problem is still necessary, our results give additional weight to the view that limiting similarity is a good starting point of biological thinking. Species packing, that is much denser than expected, seems to require quite specific model choices.

\section{Acknowledgements}

We would like to thank Christóbal López, Mats Gyllenberg, Péter Szabó and Dávid Völgyes for discussions; Peter Abrams, Olof Leimar and Annette Ostling for commenting on a previous version of the manuscript. This work was financed by OTKA grant No T049689.

\section{References}

Abrams, P. A., 1983. The theory of limiting similarity. Annual Review of Ecology and Systematics $14,359-376$

Grant, P. R., Schluter, D., 1984. Interspecific competition inferred from patterns of guild structure. In: Strong, D. R., Sinberloff, D., Abele, L. G., Thistle, A. B. (Eds.), Ecological communities: Conceptual issues and the evidence. Princeton University Press, Princeton. 
Grant, P. R., 1999. Ecology and evolution of Darwin's finches. Princeton University Press, Princeton.

Gyllenberg, M., Meszéna, G., 2005. On the impossibility of coexistence of infinitely many strategies. Journal of Mathematical Biology 50, 133-160.

MacArthur, R. H., Levins, R., 1967. The limiting similarity, convergence, and divergence of coexisting species. American Naturalist 101 (921), 377-385.

Maynard Smith, J., Szathmáry, E., 1995. The major transitions in evolution. W.H. Freeman Spektrum, Oxford.

May, R. M., MacArthur, R. H., 1972. Niche overlap as a function of environmental variability. Proceedings of the National Academy of Sciences USA 69, 1109-1113.

May, R. M., 1973. Stability and Complexity in Model Ecosystems. Princeton University Press, Princeton.

Meszéna, G., Gyllenberg, M., Pásztor, L., Metz, J. A. J., 2006. Competitive exclusion and limiting similarity: a unified theory. Theoretical Population Biology 69, 68-87.

URL http://angel.elte.hu/ geza/LimSimPre.pdf

Pigolotti, S., López, C., Hernández-García, E., Andersen, K. H., 2008. On the robustness of Gaussian competition in niche models. IFISC Publications.

URL http://www.ifisc.uib.es/publications/downfile.php?fid=3658

Pigolotti, S., López, C., Hernández-García, E., 2007. Species clustering in competitive LotkaVolterra models. Physical Review Letters 98, 258101.

Rosenzweig, M. L., 1995. Species diversity in space and time. Cambridge University Press, Cambridge. 
Roughgarden, J., 1979. Theory of population genetics and evolutionary ecology. Macmillan, New York.

Sasaki, A., Ellner, S., 1995. The evolutionarily stable phenotype distribution in a random environment. Evolution 49(2), 337-350.

Sasaki, A., 1997. Clumped distribution by neighborhood competition. Journal of Theoretical Biology $186,415-430$.

Schluter, D., 1982. Distributions of galapagos ground finches along an altitudinal gradient: the importance of food supply. Ecology 63, 1504-1517.

Szabó, P., Meszéna, G., 2006. Limiting similarity revisited. Oikos 112, 612-619.

Szilágyi, A., Meszéna, G., 2008. Two-patch model of spatial niche segregation. Evolutionary Ecology in press.

Szilágyi, A., Meszéna, G., 2009. Niche theory of sturctured populations. Journal of theoretical Biology in press.

Vandermeer, J. H., 1975. Interspecific competition: A new approach to the classical theory. Science $188,253-255$. 

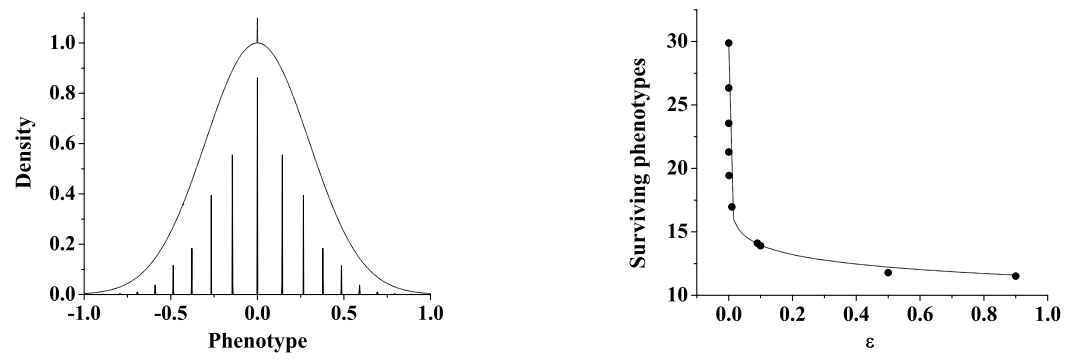

Figure 1: Perturbation with a single peak at $x=0$. Simulation parameters here and in the other figures, if not indicated otherwise: $\omega=0.3, \sigma=0.08$. Left: Equilibrium solution with perturbation amplitude $\varepsilon=0.1$; the curve represents the carrying capacity $K(x)$. Right: Number of survivors as a function of $\varepsilon$. Dots are simulation results; the curve shows the best fit with the function $m=\alpha-\beta \ln \varepsilon$. Fitting parameters: $\alpha=11.5 \pm 0.2, \beta=1.08 \pm 0.03$.
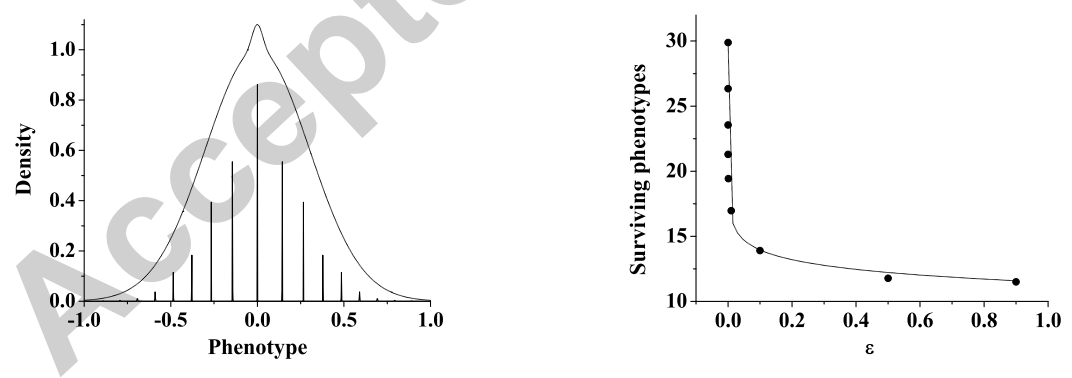

Figure 2: A similar perturbation with a smooth, Gaussian peak of width $\xi=0.03$ at $x=0$. The left pane shows the equilibrium distribution with $\varepsilon=0.1$, the right pane shows the $\varepsilon$-dependence of the number of species in equilibrium. Fitting parameters: $\alpha=11.5 \pm 0.2, \beta=1.08 \pm 0.03$, which are the same as on Fig. 1. 

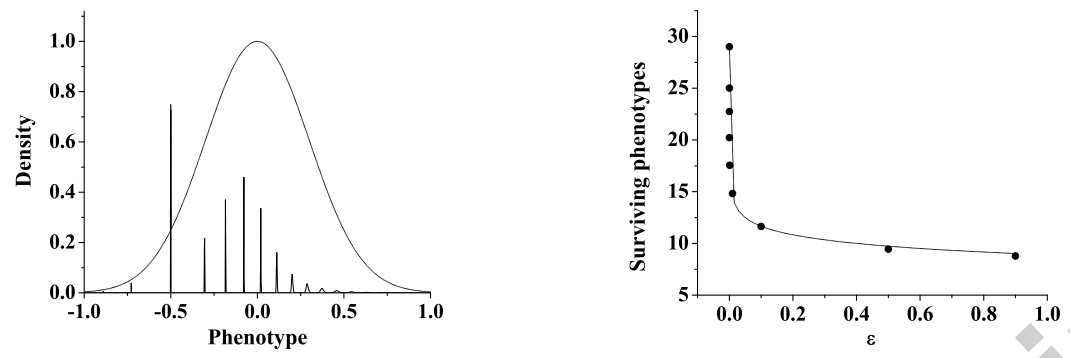

Figure 3: Perturbation with an assymmetrically located peak at $x=-0.5$. Again, the left pane is the equilibrium distribution $(\varepsilon=0.5)$, the right pane shows the $\varepsilon$-dependence. Fitting parameters: $\alpha=8.9 \pm 0.2, \beta=1.20 \pm 0.03$.
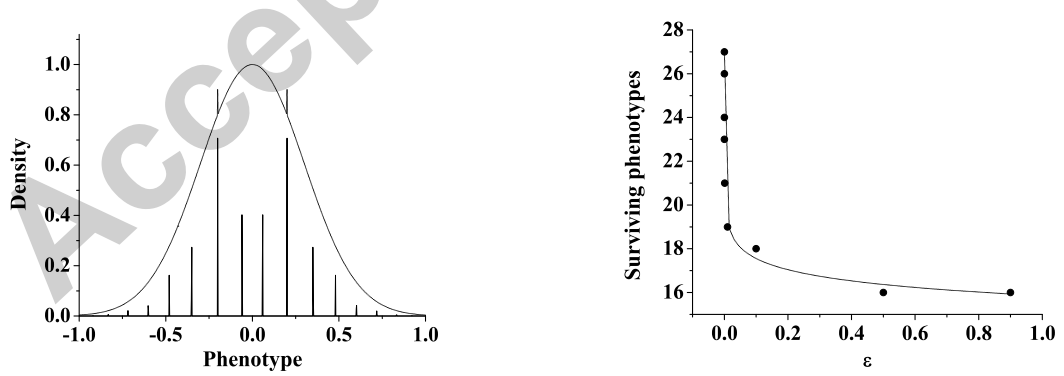

Figure 4: As before, but with a double-peaked perturbation at $x= \pm 0.2$. The example on the left pane has $\varepsilon=0.1$. Fitting parameters: $\alpha=15.86 \pm 0.19, \beta=0.738 \pm 0.027$. 



Figure 5: Perturbation with the fractal function given by Eq. (14), $\eta=1.5$. The value of $\varepsilon$ on the left is 0.01 . Fitting parameters: $\alpha=18.6 \pm 0.9, \beta=2.4 \pm 0.1$.
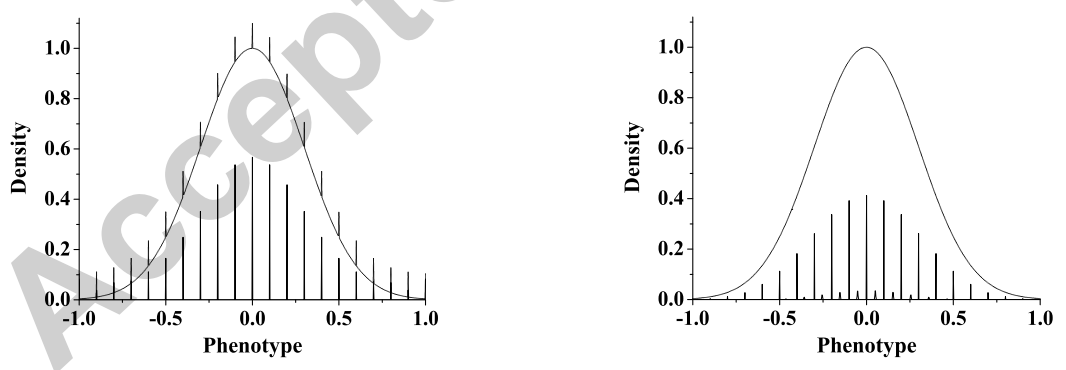

Figure 6: Equilibrium distribution for the multi-peaked perturbation of Eq. (15) with different perturbation amplitudes. Left: $\varepsilon=0.1$, right: $\varepsilon=10^{-5}$. Note the extra equilibrated species for very small perturbations. 



Figure 7: The typical distance $\Delta x=2 / \alpha$ between the neighbouring species as a function of the half niche-width $\sigma$. Fitting equation: $\Delta x=\gamma+\delta \sigma$. Left: perturbation with a single central peak; fitting parameters: $\gamma=0.002 \pm 0.002, \delta=2.13 \pm 0.02$. Right: fractal perturbation; $\gamma=0.000 \pm 0.003$, $\delta=1.30 \pm 0.05$. Observe that the fitted $\gamma=0$ represents strict proportionality. The proportionality constant $\delta$ is situation dependent but is in the order of magnitude 1 . 